\title{
Responding to the Kerr/Haslam Inquiry
}

In 2004 I was asked by the College first to respond to the Inquiry's questions and later to attend a 'stakeholders' meeting'. This was not so much in my capacity as Treasurer but as an officer with an interest in the issue of risk and professional difficulties for psychiatrists. A review of the public reports from the General Medical Council (GMC) of their determinations in the Professional Conduct Committee had made it evident that sexual misconduct was probably the single greatest cause of a finding of serious professional misconduct against a psychiatrist. In addition, I had contributed to an earlier debate on sexual safety for women in psychiatric hospitals (Subotsky, 1991, 1993).

The College was formally asked by the Inquiry for comments on its policies and views on the relevant issues in the periods 1961-1988 (during which time the abuses in York had taken place) and 1988 to the present day (to determine what changes had been made). To some extent, the response had to be speculative, especially about the earlier period. Attention was drawn to the GMC Registrar's 'Statement on abuse and harassment within psychiatric hospitals' (Gath, 1989), which noted that 'incidents involving sexual harassment of patients in psychiatric hospitals have been brought to the notice of the Public Policy Committee'. Brief recommendations were made regarding security, education of patients and training of staff; there was no specific allusion to the situation of abuse of patients by staff. This was taken further by the College's Council Report Sexual Abuse and Harassment in Psychiatric Settings (Royal College of Psychiatrists, 1996):

'It has been suggested that abuse/ harassment perpetrated by psychiatric staff involves 5-9\% of staff... [there is] continual pressure on the staff to get caught up in psychopathology. . . People may seek out employment where the vulnerable may be exploited. . . there needs to be an effective policy of vetting staff and volunteers.'

In the past few years, the GMC has notified the College of members being considered under its procedures so that appropriate action can be taken. The GMC also brought to the College's attention the fact that allegations about sexual impropriety were being made against psychiatrists and asked for the development of specific standards to facilitate judging what was appropriate and what was inappropriate, specifically in this area but also with respect to amplification of the GMC's document Good Medical Practice (General Medical Council, 2001).

The College responded with two policy documents and both were circulated to all members:

- Good Psychiatric Practice (Royal College of Psychiatrists, 2000)

- Vulnerable Patients, Vulnerable Doctors: Good Practice in our Clinical Relationships (Royal College of Psychiatrists, 2002)
Of particular relevance in Good Psychiatric Practice is the section on the 'trusting relationship' (pp.7-9): 'The psychiatrist will ... respect patients' privacy and dignity... be mindful of the vulnerability of some patients to exploitation within the therapeutic relationship'; and within the psychotherapy section, 'Good practice in psychotherapy will include... paying particular attention to boundaries, time and place, and being sensitive to the psychological implications of transgressing boundaries, e.g. through touch or self-revelation'.

Vulnerable Patients, Vulnerable Doctors clearly states in the summary (p. 23) that:

- 'Physical contact may be perceived as an appropriate comfort in some situation and as an assault in other. What matters is the meaning of the doctor's behaviour for the patient, not the innocence of the doctor's intentions.

- Relationships of sexual intimacy between doctor and patient are totally unacceptable. Both patient and doctor will be protected by the use of chaperones where misinterpretation is possible.

- Innovative techniques should be used only if there is good evidence as to their propriety and effectiveness, with the consent of the patient and with full preparation.

The book on psychosexual disorders in the College Seminar series (West, 1998) included a chapter on sexuality in mental health facilities which elaborated on the issue of sexual contact between staff and patients:

'[it] should be explicitly prohibited and defined as a serious disciplinary offence. This necessarily goes beyond what is forbidden by criminal law, which penalises intercourse with a patient only if the perpetrator is male and the patient female and is silent on the issue of consensual acts other than intercourse. . . . Complaints . . . are quite common. . . All institutions need a written policy with procedures clearly laid down. . . . A distressed victim has a right to have the incidence properly established.'

The Inquiry also wished to know about the College's complaints and disciplinary procedures and were advised that Membership could be terminated if there were erasure from the GMC register or equivalent. If a member were suspended from such a register other than on grounds of ill-health, or had been convicted of a criminal offence, or 'acted in any respect in a dishonourable or unprofessional manner' or was otherwise unfit, the case could be brought to the Court of Electors who could recommend termination of Membership, with the right of appeal to Council (Royal College of Psychiatrists, 2001). In fact, 24 members had their Membership of the College terminated between 2000 and 2005, mainly following removal from the GMC register, but also following serious criminal conviction. The Inquiry was informed that 
a complaints system was being developed and that the disciplinary procedure was being revised.

opinion

\& debate

\section{Subsequent progress}

The stakeholders' meeting was extremely informative and well run, and the chairman was keen that organisations should continue to take matters forward, not just wait for the formal recommendations. He was particularly concerned that the College should consider developing a 'Code of Ethics' (as suggested by Sarkar \& Adshead, 2003), partly to clarify what could be appropriately considered grounds for disciplinary action, and to define whether sexual relationships with 'former patients' should be permissible and if so under what circumstances. Other psychiatric associations have made this ruling, because of the special nature of the psychotherapeutic relationship even where the corresponding medical regulatory body has not required this of all doctors. The College is taking these two issues and many others through the Clinical Governance Committee, to be considered in the revision of the policy documents mentioned above.

\section{Research into prevalence}

The College has reservations about the potential usefulness of member surveys of sexualised behaviour between doctors and existing or former patients, especially since such relationships have now been made criminal, but supports the better collection and analysis of data from reporting systems, such as trust complaints and incidents, and referrals to the GMC

\section{Centralised records}

The Inquiry recommended some form of central recording linked to the named professional, which would include non-proven allegations. Although this approach has had some success in sexual offence prosecutions, the likelihood of false allegations and the suggestion of easy access to such data makes this approach problematic. Even though the GMC could potentially adopt such a system, it would not include the many mental health workers who are not professionally registered. Criminal Records Bureau checking could usefully be extended.

\section{Physical examinations and the use of chaperones}

The Clinical Governance Committee will offer further advice on this in the revised edition of Vulnerable Patients, Vulnerable Doctors (Royal College of Psychiatrsits, 2002). Different services should have unambiguous protocols about what level of physical examination is required under different circumstances (e.g. admission, first out-patient attendance, emergency, home visits). If physical examination is intended, patients should be given information and offered a chaperone.

\section{Treatments}

The Inquiry warned against the use of 'unorthodox' treatments without good explanation, consent and monitoring. Clearly, some treatments provide more opportunity for abuse than others. Risky situations include the use of frequent one-to-one sessions in isolated locations and out of hours, the use of benzodiazepines and other sedatives, hypnotherapy, and 'touching' treatments, such as massage or aromatherapy. It recommended that trusts should have evidence bases and protocols for treatments and that they should be aware of therapies being undertaken by all staff, through appraisals and job plans. A register of treatments would be useful for recording treatments and approved protocols, and could include chaperone need, level of training and supervision requirements.

\section{Recruitment practices}

Previous formal inquiries suggested that a number of sexual abuse incidents could be prevented if good recruitment practices were followed and if agency and non-clinical staff were also vetted. The Kerr/Haslam Inquiry recommended that one of the referees in any job application should be the consultant who conducts the applicant's appraisal, their clinical director or their medical director, and that references should be obtained from the three most recent employers and should be 'properly checked'. The College will need to ensure that this is part of the training and advice given to College appointments advisers.

\section{Staff performance}

Although job plans and appraisal systems are in place, they are not always optimally utilised. A '360 degree' appraisal would be helpful and serious consideration should also be given to ensuring clinical supervision systems for senior clinicians. Some trusts do this with continuing professional development but other models would be possible.

\section{Training}

The Inquiry recommends that health workers at all levels should be able to discuss feelings of sexual attraction 'without the automatic risk of disciplinary action' and that boundary issues should be part of the curriculum. The Psychotherapy Faculty is developing further ideas on this and the topic will be included in the next annual meeting. A useful review on the topic of professional abuse of patients was provided by Sarkar (2004).

\section{College policy}

The Inquiry had hoped that the College might act as a separate regulatory body, with compulsory Membership and complaints and sanction systems. The College view 
has been that Membership or affiliation to the College should be broadened as widely as possible and trusts encouraged to employ staff with professional memberships. Because there are already many other pathways for taking action against poorly performing doctors, which can lead to confusion, the disciplinary procedure has been revised to focus on College-related activities and a complaints policy has been developed. Meetings have been held with the GMC and the National Clinical Advisory Service (NCAS) to clarify respective roles and expectations if complaints are received, whether from patients or professionals.

There are evident difficulties for professionals who are potential 'proxy complainants' or whistle-blowers. The GMC's advice on this has been considerably amplified, but difficulties in reporting suspicions about a senior figure remain, especially if the patient has not given consent. The College would be supportive of any member in this situation through its new member support system.

\section{Patient information and support}

The College supports the recommendations on providing patients with information on what to expect in assessment and treatment, how to make complaints, and sources of independent advocacy and support. The increasing involvement of users and carers within the College could facilitate the development of appropriate leaflets, although the responsibility is local. Later access to psychological counselling or psychiatric treatment for abused patients may be difficult to obtain because of fear of recrimination, long waiting lists or lack of funding. The voluntary agency 'The Prevention of Professional Abuse Network' has considerable experience in this field and its expertise could be utilised in providing patient information and in training.

\section{Other issues}

The issues of sexual abuse by doctors brought to light by this Inquiry and others have not received much professional attention in the UK - whereas there has been much more debate and action in North America, Australia and New Zealand. The psychiatrists whose behaviour was the focus of the Inquiry were senior and apparently 'untouchable' for many years. Little emphasised was the fact that many allegations came from nurses who were also their patients - this dual relationship apparently made taking action more difficult rather than easier. This situation should perhaps be taken up by the College with both the Royal College of Nursing and occupational health physicians. Furthermore, there were suggestions that the two doctors may at least on some occasions have covered each other, again a situation known to occur with other forms of sexual and institutional abuse. Policies alone can support although not ensure change, but it is undoubtedly time to open up the debate.

\section{Declaration of interest.}

None.

\section{References}

GATH, A. (1989) Statement on abuse and harassment within psychiatric hospitals. Psychiatric Bulletin, 13, 460 GENERAL MEDICAL COUNCIL (2001) Good Medical Practice. London: GMC.

ROYAL COLLEGE OF PSYCHIATRISTS (1996) Sexual Abuse and Harassment in Psychiatric Settings (Council Report CR52). London: Royal College of Psychiatrists.

ROYAL COLLEGE OF PSYCHIATRISTS

(2000) Good Psychiatric Practice (Council Report CR83). London: Royal College of Psychiatrists.

ROYAL COLLEGE OF PSYCHIATRISTS (2001) Supplemental Charter, By-Laws and Regulations (Occasional Paper OP52). London: Royal College of Psychiatrists.

ROYAL COLLEGE OF PSYCHIATRISTS (2002) Vulnerable Patients, Vulnerable Doctors: Good Practice in our Clinical Relationships (Council Report CR101). London: Royal College SARKAR, S. P. (2004) Boundary violation and sexual exploitation in psychiatry and psychotherapy: a review. Advances in Psychiatric Treatment, 10, 312-320.

SARKAR, S. P. \& ADSHEAD, G. (2003) Protecting altruism: a call for a code of ethics in British psychiatry. British Journal of Psychiatry, 183, 95-97.

SUBOTSKY, F. (1991) Issues for women in the development of mental health services. British Journal of Psychiatry, 158 (suppl. 10), 17-21.

SUBOTSKY, F. (1993) Sexual abuse in psychiatric hospitals: developing policies to aid prevention. Psychiatric Bulletin, 17, 274-276.

WEST, D. J. (1998) Problems of sexuality among people in mental health facilities. In Seminars in Psychosexual Disorders (eds H. Freeman, I. Pullen, G. Stein, et al), pp.124-141. London:

Gaskell. opinion \& debate
Fiona Subotsky Emeritus Child and Adolescent Psychiatrist, South London and Maudsley NHS Trust and HonoraryTreasurer, Royal College of Psychiatrists, e-mail: f-subotsky@rcpsych.ac.uk 